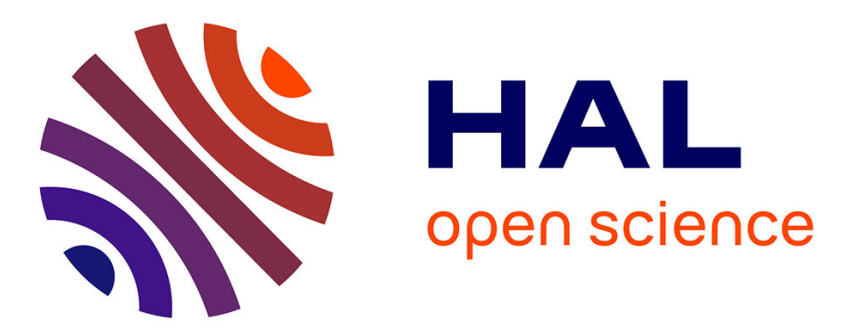

\title{
Modeling analysis of tidal bore formation in convergent estuaries
}

Andrea Gilberto Filippini, Luca Arpaia, Philippe Bonneton, Mario Ricchiuto

\section{To cite this version:}

Andrea Gilberto Filippini, Luca Arpaia, Philippe Bonneton, Mario Ricchiuto. Modeling analysis of tidal bore formation in convergent estuaries. European Journal of Mechanics - B/Fluids, 2018, 10.1016/j.euromechflu.2018.01.001 . hal-01698295

\section{HAL Id: hal-01698295 \\ https://hal.inria.fr/hal-01698295}

Submitted on 30 Oct 2018

HAL is a multi-disciplinary open access archive for the deposit and dissemination of scientific research documents, whether they are published or not. The documents may come from teaching and research institutions in France or abroad, or from public or private research centers.
L'archive ouverte pluridisciplinaire HAL, est destinée au dépôt et à la diffusion de documents scientifiques de niveau recherche, publiés ou non, émanant des établissements d'enseignement et de recherche français ou étrangers, des laboratoires publics ou privés. 


\title{
Modeling analysis of tidal bore formation in convergent estuaries
}

\author{
Andrea Gilberto Filippini (Corresponding author) \\ Team CARDAMOM, Inria Bordeaux sud-Ouest and IMB, - 200 av. de la vieille tour, \\ 33405 Talence cedex, France \\ Luca Arpaia \\ Team CARDAMOM, Inria Bordeaux sud-Ouest and IMB, - 200 av. de la vieille tour, \\ 33405 Talence cedex, France \\ Philippe Bonneton \\ CNRS, UMR EPOC, University of Bordeaux, Allée Geoffroy Saint-Hilaire, 33615 Pessac \\ cedex, France \\ Mario Ricchiuto \\ Team CARDAMOM, Inria Bordeaux sud-Ouest and IMB, - 200 av. de la vieille tour, \\ 33405 Talence cedex, France
}

\begin{abstract}
Despite the recognized impact of tidal bores on estuarine ecosystems, the large scale mechanism of bore formation in convergent alluvial estuaries is still under investigation. So far, field data exist only for a small number of estuaries, while numerical simulations employ the shallow water equations mainly focusing on the small-scale and local processes. In this work, firstly we apply the fully nonlinear weakly dispersive Serre-Green-Naghdi equations to simulate the tide propagation in a convergent estuary of idealized form, verifying that the local dispersion effects, responsible for the appearance of the secondary waves, do not influence the tidal bore onset, which only results from the large scale processes of amplification/damping and distortion of the incoming wave. In
\end{abstract}

Email addresses: andrea.filippini@inria.fr (Andrea Gilberto Filippini (Corresponding author)), luca.arpaia@inria.fr (Luca Arpaia),

p.bonneton@epoc.u-bordeaux1.fr (Philippe Bonneton), mario.ricchiuto@inria.fr (Mario Ricchiuto)

Preprint submitted to European Journal of Mechanics - B/Fluids

November 13, 2017 
a second part, we numerically investigate (225 runs) the estuarine parameter space in order to identify the physical conditions that lead to tidal bore generation. In this parameter space, we determine a critical curve which divides estuaries according to tidal bore occurrence. As a result of this investigation we have shown that bore formation is controlled by the competition between two physical processes: a) the knee-shaped distortion of the tidal wave, with flood dominance and eventually bore inception; b) the dissipation of the tidal wave, which is unfavorable to bore formation. We also provide evidence that amplification due to topographic convergence is not a necessary condition for tidal bore generation and that there exist estuaries which display both wave damping and bore development. Finally, the validity of the results has been also assessed in the presence of freshwater river discharge, showing that for low river discharge, its effect on estuarine dynamics can be neglected.

Keywords: Estuarine hydrodynamics; long wave; tidal bore formation; dispersion; undular tidal bore; Serre-Green-Naghdi equations.

\section{Introduction}

Tidal wave transformation in convergent alluvial estuaries plays a crucial role in the development of a sustainable management of water resources. For this reason, and to better understand the human impact on the estuarine ecosystem, it has been the subject of intensive scientific research. Parametric studies conducted in [1, 2, 3, 4, 5, have shown that, when neglecting river discharge effects, the estuarine hydrodynamics is controlled by only three dimensionless parameters. These parameters represent a combination of the properties of the tidal forcing at the estuary mouth (wave amplitude and period), the large-scale geometrical characteristics of the channel and the friction coefficient. For particular conditions of the above dimensional variables, of freshwater flow and of river channel bathymetry, the tidal wave may result strongly distorted when the flow turn to rise, and a bore can be observed at the beginning of the flood tide. In the lower part of the estuary, tidal wave propagation can be well described by 
the so called Saint Venant or Nonlinear Shallow Water (NLSW) equations with friction ( $c f .6$, 6 ). However the onset of a tidal bore and its evolution upstream is controlled by non-hydrostatic dispersive mechanisms ( $c f$. [7]). Even if Madsen et al. 8] and Pan and Liu [9] have shown that the non-dispersive NLSW equations with shock capturing methods can still be used to simulate breaking bore propagation with relatively good results, undular bores requires the use of other long wave modeling approaches. If weakly dispersive weakly nonlinear Boussinesq-type equations can be adopted to describe the onset of the tidal bore (cf. [7]), the nonlinear evolution of high-intensity bores requests fully nonlinear equations to be employed, as for example the Serre-Green-Naghdi (SGN) ones (cf. [10, 11, 12]). The first part of this work is thus devoted to reproduce the progressive formation of an undular tidal bore inside an idealized estuary using the SGN equations.

The results obtained in this first part will then justify the use of the NLSW model to investigate at large scales how the three dimensionless parameters, which control the estuarine long scale dynamics, influence the physical processes of amplification/damping and distortion which may lead to the bore onset. These processes take place at large spatial and temporal scales, where the non-hydrostatic effects associated with tidal bores can be neglected. Some numerical studies already used the NLSW system to simulate the propagation of tidal waves in rivers up to bore formation, but they are limited to a single [8, 9] or to a small number [4] of estuaries. A similar limitation concerns also field data: since every estuary in nature represents a unique combination of the three dimensionless variables, we dispose only of a limited number of points in the parameter space coming from in situ measurements. Based on the set of data available, it is very difficult to understand how such parameters influence the bore development process. In this paper, we bypass this major constraint through a numerical investigation of the whole space of dimensionless variables. This allows us both to characterize the global conditions for tidal bore occurrence and to analyze for each estuarine regime the nonlinear processes associated with tidal wave transformation. Our approach is based on a scaling analysis in- 
troduced in [13, where the reader can also find a collection of estuary data, which are used here to validate our numerical investigation.

The paper is structured as follows: section 2 introduces the idealized geometry used to accomplish our study and defines the dimensionless parameters emerging from the scaling analysis of the governing equations; section 3 gives some details about the solution strategy adopted to solve the SGN system, the implemented numerical scheme and motivates the choice of the type of boundary conditions used; section 4 introduces a criterion for bore detection based on experimental observation in situ; the numerical simulation of the onset of an undular tidal bore inside an idealized estuary is presented in section 5 section 6 discusses the numerical investigation on the favorable conditions for tidal bore occurrence, introducing a criterion for bore detection based on experimental observation in situ and showing the main findings of the study; finally, section 7 treats the effects of water river discharge on tidal bore formation.

\section{Problem setting}

The study of tidal wave propagation in funnel shaped estuaries is often performed under several simplifying assumptions. The geometry of real alluvial estuaries in coastal plains can be well-approximated by an exponentially decreasing width variation and flat bathymetry [14, 15, 6]. This morphology is the natural result of a morphodynamic equilibrium of the erosion/sedimentation process. For this reason, we will perform our study on the simplified case (figure 1) of a tide propagating in an idealized convergent channel of constant depth $D_{0}$ and an exponentially decreasing width $B(x)$, varying in the longitudinal direction $x$ with the law:

$$
B(x)=B_{0} e^{-x / L_{b}}
$$

where $L_{b}$ represents the convergence length of the channel. We assume a rectangular cross-section suitable, as a first approximation, to describe the behavior of a real section with the same area. 

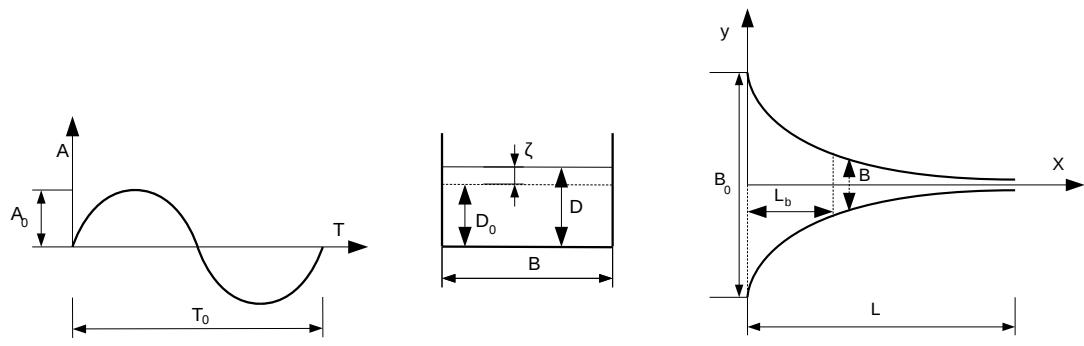

Figure 1: Sketch of the idealized geometry of the channel and basic notations.

It is a common practice in the literature to use quasi-one-dimensional systems of equations to investigate the large scale dynamics of long waves propagating in convergent channels [1, 2, 16, 5, 4,. In the case of open channels with exponentially decreasing cross section areas, the equations assume the following form:

$$
\begin{array}{r}
\frac{\partial \zeta}{\partial t}+u \frac{\partial \zeta}{\partial x}+D \frac{\partial u}{\partial x}-\frac{u D}{L_{b}}=0 \\
\frac{\partial u}{\partial t}+u \frac{\partial u}{\partial x}+g \frac{\partial \zeta}{\partial x}+C_{f 0} \frac{u|u|}{D}=0
\end{array}
$$

where $D=\zeta+D_{0}$ denotes the total water depth, with $\zeta$ the free surface elevation and $D_{0}$ the still water depth and $u$ stands for the cross-sectionally averaged flow velocity. The friction term is modeled by a quadratic law, with $C_{f 0}$ the friction coefficient.

As already remarked by others in the literature [1, 2, 4, 13, three external dimensionless parameters appears to fully control the system (2). Following the scaling of the equations proposed by Bonneton et al. [13, these parameters are:

$$
\epsilon_{0}=\frac{A_{0}}{D_{0}}, \quad \delta_{0}=\frac{L_{w 0}}{L_{b}}, \quad \phi_{0}=\frac{C_{f 0}\left(g D_{0}\right)^{1 / 2}}{\omega_{0} D_{0}} ;
$$

where $D_{0}, A_{0}$ and $\omega_{0}$ form a set of reference external parameters, respectively the water depth, the amplitude and the angular frequency of the tidal wave. Here $\epsilon_{0}$ represents the standard nonlinearity parameter, $\delta_{0}$ is the convergence ratio, $\phi_{0}$ is a friction parameter and $L_{w 0}=\left(g D_{0}\right)^{1 / 2} \omega_{0}^{-1}$ is the frictionless tidal- 
wave length scale.

Field observations reported in [3] show that in tidal bore estuaries the tidal wave nonlinearity is mainly characterized by the dimensionless parameter

$$
D_{i}^{*}=\epsilon_{0} \phi_{0} / \delta_{0}
$$

being always one order of magnitude greater than $\epsilon_{0}$.

Both theoretical studies [1] and experimental observations [3] agree that large values of the dissipation parameter $D_{i}^{*}$ produce great distortion and peaking of the free surface and velocity profiles of the tidal wave, leading to flood dominance; characteristics which correspond to necessary conditions for tidal bore formation $\left(D_{i}^{*} \geq 1.7\right.$ in $\left.[3]\right)$. However, large values of $D_{i}^{*}$ correspond also to high energy dissipation, leading to tidal damping; unfavorable to tidal bore formation. For this reason some natural estuaries, despite having high values of $D_{i}^{*}$, don't display a bore. In order to evaluate the relative importance of friction in the momentum balance, several definitions of the friction parameter $\phi_{0}$ have been introduced in the literature [1, 2, 17, 4. Although all of these definitions are analogous from a physical point of view, only the one introduced by the present authors in 13 allows to investigate separately the opposite effects of peaking and dissipation taking place for high values of $D_{i}^{*}$. In this paper we describe the details of the numerical investigation behind the scaling proposed in [13]. We will thus explore numerically the space of the external parameters $\epsilon_{0}, \delta_{0}$ and $\phi_{0}$, quantifying which range of values is in favour of tidal bore formation.

Considering that, for most alluvial estuaries, the convergence ratio $\delta_{0}$ is close to 2 (as showed in figure 2 of [13]), we have chosen to perform our analysis using a constant value $\delta_{0}=2$. Due to such a simplification, the expression of the dissipation parameter (4) reduces to: $D_{i}^{*}=\alpha \epsilon_{0} \phi_{0}$ (with $\alpha$ constant). Thus, it is possible to investigate the separate effects of peaking and dissipation, both contained in $D_{i}^{*}$, by numerically exploring only the plane of the dimensionless parameters $\left(\phi_{0}, \epsilon_{0}\right)$. The effects of nonlinear dispersion and of the discharge are also investigated, while we leave out for the moment the influence of bathymetric 
variations.

\section{Numerical model}

\subsection{Governing equations}

For nonlinear long waves, a reasonably general description is provided by the fully nonlinear weakly dispersive Serre-Green-Naghdi equations. As already mentioned, this model allows to simulate the phenomenon in its entirety, including the nonlinear dispersive effects active on the smaller scales. To the authors' knowledge, it does not exist so far any mathematical model allowing to account for such effects in quasi-1D setting. The closest work is the very recent paper of Winckler and Liu [18, who developed a set of weakly nonlinear Boussinesq equations. This justifies the use of a full two-dimensional model. In particular, the SGN equations can be recast as [19, 20]:

$$
\begin{aligned}
\frac{\partial \zeta}{\partial t}+\nabla \cdot(D \boldsymbol{u}) & =0 \\
\frac{\partial u}{\partial t}+(\boldsymbol{u} \cdot \nabla) \boldsymbol{u}+g \nabla \zeta+C_{f 0} \frac{\boldsymbol{u}|\boldsymbol{u}|}{D} & =\boldsymbol{\psi}, \\
(\mathrm{I}+\mathrm{T}) \boldsymbol{\psi} & =\mathrm{T}\left(g \nabla \zeta-C_{f 0} \frac{\boldsymbol{u}|\boldsymbol{u}|}{D}\right)-\mathrm{Q}(\boldsymbol{u})
\end{aligned}
$$

where $\boldsymbol{u}$ is the depth averaged velocity vector of horizontal component $u$ and transversal one $v, \boldsymbol{\psi}$ characterizes the non-hydrostatic and dispersive effects, and the linear operators $\mathrm{T}(\cdot)$ and $\mathrm{Q}(\cdot)$ are the same defined in [20] and their expressions are given in appendix A.

It is worth noting that, when applying the scaling proposed in [13] and recalled in section 2 to system (5) (details are reported in appendix A), an additional dimensionless parameter $\mu^{2}=\left(D_{0} / L_{0}\right)^{2}$ appears into the equations, multiplying all the dispersive terms ( $c f$. equation $(17 \mathrm{~b})$ ). This parameter is responsible for the fact that the non-hydrostatic effects become negligible when the characteristic scale of the phenomenon is large, transforming the original SGN system of equations into the non-conservative form of the Non-Linear Shallow Water (NLSW) system. For this reason, dispersion does not influence significantly 
the large scales of tidal wave propagation, which can be well described by the NLSW equations. A direct verification of this is given and discussed in section 5 .

\subsection{Numerical strategy}

To solve system (5) we adopt the strategy illustrated in [20]. Given an initial solution, the Serre-Green-Naghdi system is solved in two independent steps. First, the elliptic equation $5 \mathrm{c}$ is solved for the non-hydrostatic term $\psi$. Then, an hyperbolic step is performed for equations (5a)-(5b), evolving the flow variables in time. This strategy solution has been shown to be very flexible and robust, producing accurate results with different combinations of discretization schemes in space and time. For this study, in the hyperbolic phase a third order MUSCL finite volume scheme has been used, together with a third order SSP Runge-Kutta method for the evolution in time. The elliptic phase is solved, instead, with a classical second order finite element approach. The overall hybrid FV-FE scheme obtained is thus characterized by a dispersion error of the same order, or smaller, than those produced by fourth order finite differencing, providing that at least third order of accuracy is guaranteed for the hyperbolic component. The interested reader can refer to [20] for more details.

\subsection{Boundary conditions}

The choice of the boundary conditions affects the flow field significantly [21]. While exact boundary conditions can be generally imposed in the case of homogeneous hyperbolic problems, this remains an active research topic when source terms are included inside the equations. The task becomes even harder working with dispersive models. Therefore, while the fully reflective wall boundary condition can be obtained by imposing some symmetric conditions stemming from the satisfaction of : $\mathbf{u} \cdot \mathbf{n}=0$ on the wall line (being $\mathbf{n}$ the normal direction to the wall), inflow and outflow boundaries require more complex treatments ( $c f$. [22, 23, 24, 25]). 
In this work, the interest in long scale dynamics justifies the use of the NLSW invariants also when the SGN equations are solved inside the computational domain [26]. The illustrative results contained in section 5 show that the effects of this approximation on the large scale is completely negligible on both coarse and fine computational meshes.

In practice, the seaward boundary condition is applied by imposing in the incoming NLSW Riemann invariant the free surface given by the sea level tidal oscillation:

$$
\zeta(t)=A_{0} \sin \left(\frac{2 \pi t}{T_{0}}\right)
$$

We consider here the case of semi-diurnal tides with period $T_{0}=12.41[h]$.

On the landward boundary, the outflow condition is set imposing the information of still water (we recall that the river discharge is neglected at this level) coming from far on the right. However, the imposition of the homogeneous invariant cannot take into account the rise of the mean (tidally averaged) water level landward caused by the friction ( $c f$. [5]), introducing a non-physical behavior of the solution in the outlet proximity. For this reason, the outflow boundary conditions has been coupled with an extension of the computational domain, to reduce the spurious influences on the solution in the region of study. In practice it has been enlarged up to $x=6 L_{b}$, to further limit non-physical effects due to the boundary condition.

In order to guarantee the reliability of the results, a sensitivity study has been performed in comparison with the technique of boundary imposition applied by [21] and a reference solution computed using a very long computational domain. For the interested reader the results of such study are reported in appendix B.

\section{Computations setting}

Tide propagation up to an estuary is, in nature, partly limited by an increasing bottom slope and by discharge. Considering that the effects of both the bathymetric variations and the river discharge have been, at first, neglected 

$L_{c}=3 L_{b}$.

\begin{tabular}{|l|c|c|c|}
\hline Estuaries & $L_{b}[\mathrm{~km}]$ & $x_{c}[\mathrm{~km}]$ & $x_{c} / L_{b}$ \\
\hline Gironde/Garonne & 43 & 90 & 2.09 \\
Hooghly & 25 & 60 & 2.4 \\
Humber & 25 & 75 & 3.0 \\
Pungue & 17 & 50 & 2.94 \\
Qiantang & 40 & 90 & 2.25 \\
Severn & 41 & 55 & 1.34 \\
\hline
\end{tabular}

Table 1: Ratio between the location of tidal bore inception $x_{c}$ and convergence length $L_{b}$ for some alluvial estuaries. Data taken from [3].

in our simulations, even the weakest and linear tidal wave would be able to propagate landward and, being dissipated by only friction, it would reaches non-physical distances with also possible generation of unnatural bores. For this reason, and for later use, a characteristic physical length $L_{c}$ has been introduced to limit the region in which the tidal signal is assumed to be physically relevant. In particular, examining real estuaries data, we observed that tidal bores occur, before reaching a distance of $3 L_{b}$ from the estuary mouth, see table 1. Thus, we have chosen to limit the region of interest for our simulations to

Moreover, tidal bore inception is a continuous process which takes place as a gradual increase of the free surface slope at the beginning of the flood phase. The same continuous increase of steepness is observed in our simulations. A criterion is thus needed to detect the bore onset, within the domain $\left[0, L_{c}\right]$ just defined. During the two measurement campaigns on the Garonne river, Bonneton et al. 3 observed that bore passage was associated to an increase in the value of the free surface spatial gradient, at the start of the flood, with at least one order of magnitude (from $\mathscr{O}\left(10^{-4}\right)$ in the smooth case without tidal bore). For each numerical simulation we compute, in a post-processing phase, the spatial slope of the free surface all along the domain, up to $L_{c}$, retaining for each position $x$ the maximum value in the tidal period. The quantity $A_{\max }$ has 
been defined as:

$$
A_{\text {max }}=\max _{x \in L_{c}}\left[\max _{t \in T_{0}}\left(\frac{\partial \zeta(x, t)}{\partial x}\right)\right]
$$

and the following criterion to determine tidal bore onset is used: $A_{\max } \geq 10^{-3}$.

\section{Undular tidal bore formation}

Preliminary simulations concerning the development of an undular tidal bore into the idealized channel of figure 1 are reported in this section. We consider a strongly nonlinear and weakly dissipative channel of $\epsilon_{0}=0.7$ and $\phi_{0}=10$. To visualize the results we consider pointwise plots and distributions of the relevant quantities along the channel axis. In developed flow conditions, deviations in the transversal direction have been quantified to be less than $1.5 \%$.

Figure 2 shows the time series over one tidal cycle of the computed free surface elevation, measured at 11 gauges positions along the channel. It appears evident that the results provided by the SGN model do not differ respect to those obtained by using the NLSW model. Differences appear, instead, focusing on the region of the shock formation. The results obtained are then reported in figure 3 and show the formation process of a undular tidal bore. Also in this case, the two models give identical results in the whole domain except when the shock is formed ( $c f$. top of figure 3). On the discontinuity, in fact, the SGN model provides an undular bore with local formation of a train of secondary waves. These waves are characterized by a wavelength of $50[\mathrm{~m}]$ and a period of $6[s]$ ( $c f$. bottom of figure 3). However, it can be remarked that the position of the bore inception, the mean bore jump and its celerity are well-described by the NLSW model. This justifies its application for investigations on the large scale estuary dynamics. Studies concerning the local effects on tidal bores or flooding problems cannot, instead, avoid the use of a dispersive model to correctly reproduce the peaks of water height due to the secondary waves.

Field observations lead Bonneton et al. 3] to consider that the location of the tidal bore onset is associated with the appearance of an inflexion point on the 


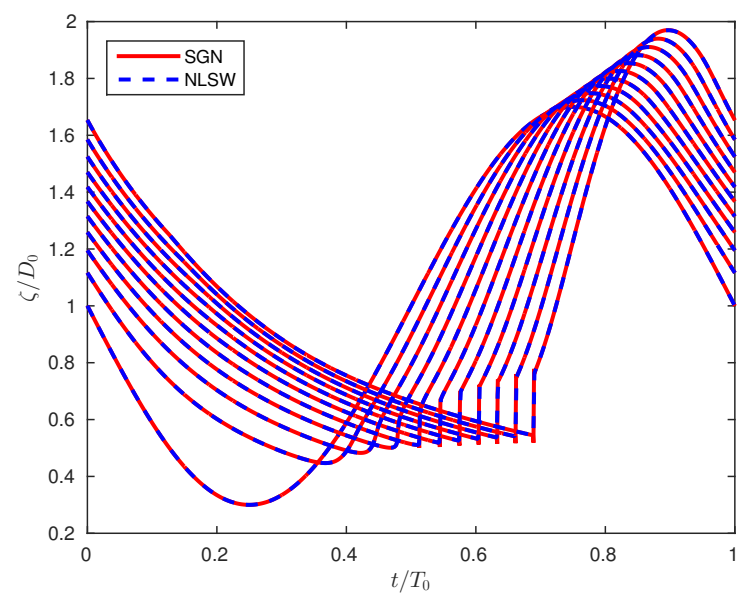

Figure 2: Illustrative result of a tidal propagation into an idealized channel with the shape described in section $2 \epsilon_{0}=0.7, \phi_{0}=10$. Computed free surface profile, measured at 11 stations along the channel corresponding to $x=\alpha L_{B}$ with $\alpha=0: 1 / 3: 3$. The simulation have been realized using both the NLSW and the SGN models.

tidal wave profile. However, these authors stress the difficulty to characterize the tidal bore onset on the river, since this would require a high density of water elevation measurements over a long distance. By contrast, with our numerical simulations we are able to provide a full set of subsequent snapshots ( $c f$. figure (4), describing the progressive transition from a tidal wave regime without bore ( $c f$. figures $4 \mathrm{a}$ and $4 \mathrm{~b}$ ) to a well-developed undular tidal bore regime ( $c f$. figures $4 \mathrm{~g}$ and $4 \mathrm{~h}$. The gradual evolution of the surface profile towards the bore inception passes by the appearance of an inflexion point at the beginning of the rising tide.

In the middle and upper part of the estuary, the value of $A_{\max }$, used in our tidal bore onset criterion, is registered at the beginning of the rising tide. As a consequence, the pictures of figure 4 allow to observe the temporal evolution of such quantity as the tide propagates landward. The values of $\max _{x \in L_{c}}\left(\frac{\partial \zeta}{\partial x}\right)$ for the eight pictures of figure 4 are, thus, showed in table 2 for the two NLSW and SGN model. It is worth to note that the dispersive terms becomes relevant after the threshold of $A_{\max }$ has been reached, i.e. when tidal bore occurrence has 

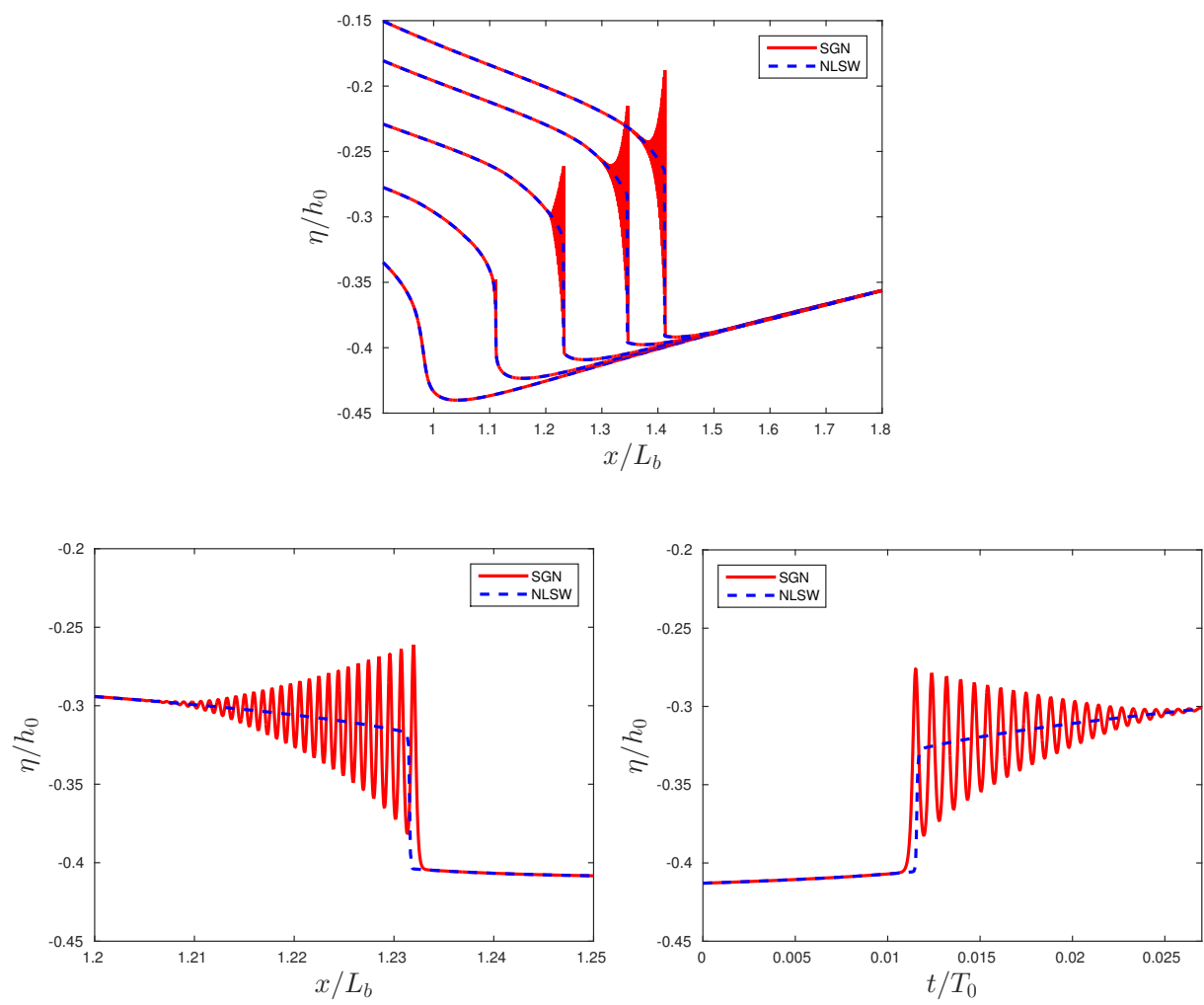

Figure 3: Illustrative result of tidal propagation into an idealized channel with the shape described in section 2 $\epsilon_{0}=0.7, \phi_{0}=10$. Top: computed free surface profile at different increasing times of the simulation. Bottom-left: free surface signal in space. Bottom-right: free surface signal in time.

already been flagged by our criterion. We can, thus, conclude that tidal bore appearance is completely determined by the values of the external parameters $\epsilon_{0}, \delta_{0}$ and $\phi_{0}$ and is not influenced by the choice of the model used for the numerical simulations.

\section{Study of tidal bore occurrence}

The results of the previous section show that dispersive effects are not necessary to study the large scales of the propagation and transformation of the tide. At these scales the NLSW system is a sufficiently accurate and more efficient 


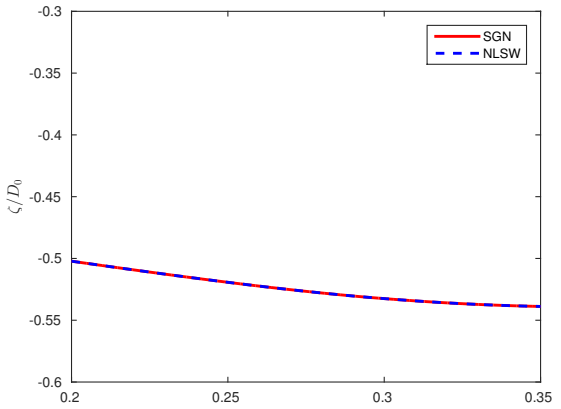

(a)

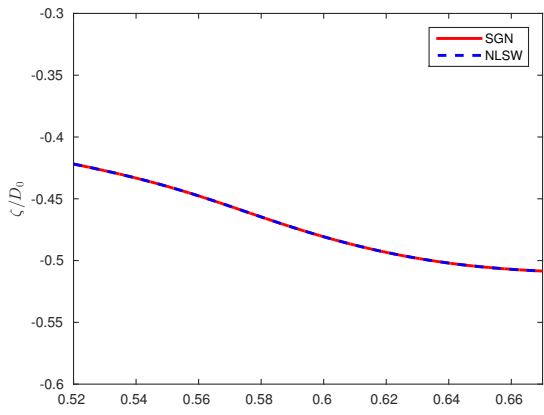

(c)

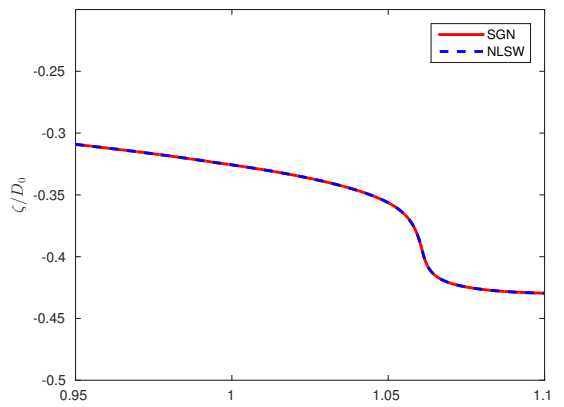

(e)

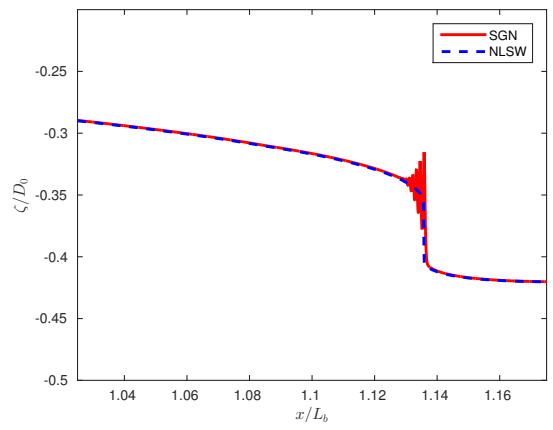

(g)

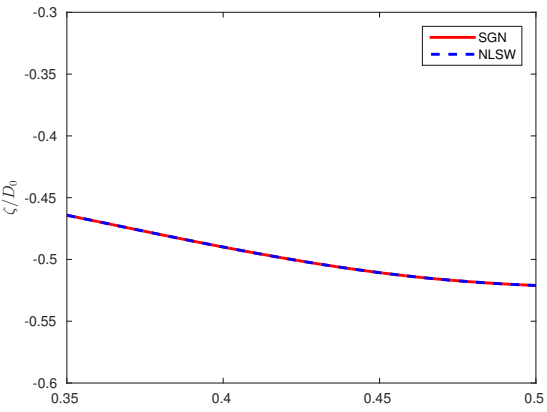

(b)

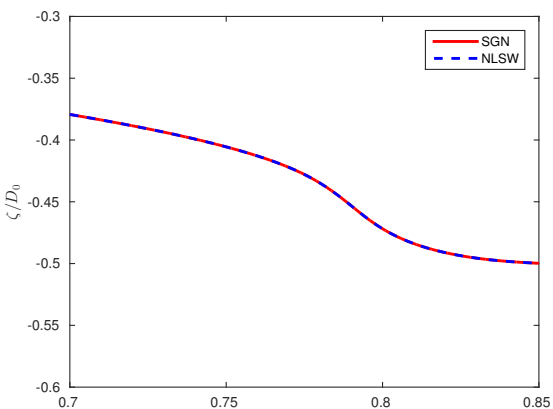

(d)

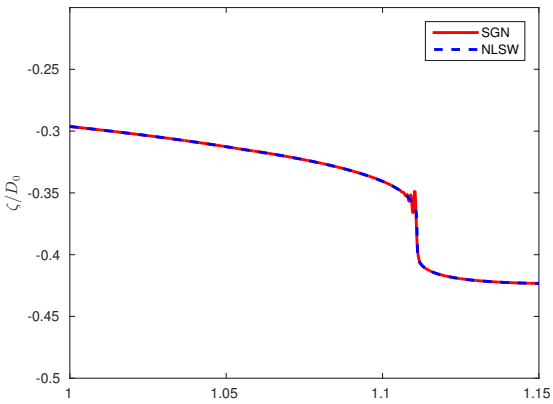

(f)

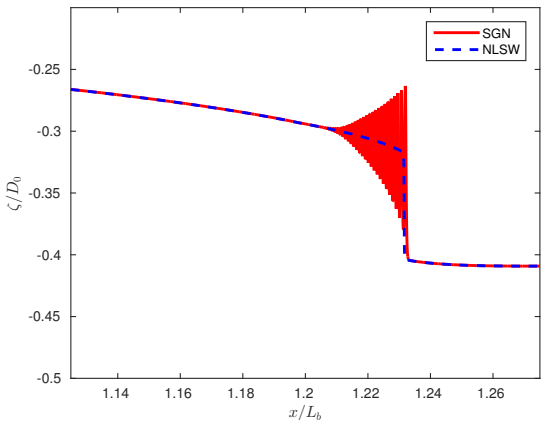

(h)

Figure 4: Computed free surface profile at different increasing times of the simulation, showing the undular bore inception from the formation of an inflexion point of the wave profile: from top left to bottom right. 


\begin{tabular}{|c|c|c|}
\hline & NLSW & SGN \\
\hline a) & $7.25 \times 10^{-5}$ & $7.25 \times 10^{-5}$ \\
b) & $1.05 \times 10^{-4}$ & $1.05 \times 10^{-4}$ \\
c) & $1,74 \times 10^{-4}$ & $1,74 \times 10^{-4}$ \\
d) & $4.11 \times 10^{-4}$ & $4.11 \times 10^{-4}$ \\
\hline e) & $\mathbf{1 . 9} \times \mathbf{1 0}^{-\mathbf{3}}$ & $\mathbf{1 . 9} \times \mathbf{1 0}^{-\mathbf{3}}$ \\
f) & $\mathbf{2 . 8 7 \times \mathbf { 1 0 } ^ { - 3 }}$ & $\mathbf{1 . 6 1} \times \mathbf{1 0}^{-\mathbf{3}}$ \\
g) & $\mathbf{5 . 0 4} \times \mathbf{1 0}^{-\mathbf{3}}$ & $\mathbf{3 . 7 7} \times \mathbf{1 0}^{-\mathbf{3}}$ \\
h) & $\mathbf{9 . 4 7} \times \mathbf{1 0}^{-\mathbf{3}}$ & $\mathbf{7 . 3 8} \times \mathbf{1 0}^{-\mathbf{3}}$ \\
\hline
\end{tabular}

Table 2: Values assumed by the quantity $\max _{x \in L_{c}}\left(\frac{\partial \zeta}{\partial x}\right)$ in the several pictures of figure 4 and for the two NLSW and SGN models. Bold numbers represent values which satisfy the tidal bore onset criterion: $A_{\max } \geq 10^{-3}$

model. This is why we will employ it here to perform the numerical investigation of the space $\left(\phi_{0}, \epsilon_{0}\right)$ of dimensionless parameters, trying to quantify the favorable conditions for bore inception. However, dispersion simulations will still be used in some cases to provide some quantification of the local order of magnitude of the non hydrostatic terms. This quantitative comparison will allow to comfort our hypothesis and provide further insight into the mechanisms of bore formation.

\subsection{Investigation of the plane $\left(\phi_{0}, \epsilon_{0}\right)$}

We have performed 225 simulations of the idealized case of study defined in section 2, corresponding to an equivalent number of estuaries. We recall that the value of convergence ratio is constant, $\delta_{0}=2$, and thus each simulation represents a unique combination of the parameters $\epsilon_{0}, \phi_{0}$, corresponding to precise conditions of the tidal forcing at the mouth and to specific geometrical and physical properties of the channel. In such a way we have systematically investigated the plane $\left(\phi_{0}, \epsilon_{0}\right)$, applying the criterion described in section 4 in 
order to detect bore formation. Figure 5(left) shows the contour lines of the quantity $A_{\max }$ in the plane of the parameters $\left(\phi_{0}, \epsilon_{0}\right)$, performed by collecting and linearly interpolating the simulations' results. The red color denotes the region where the bore detection criterion is satisfied, while the blue one represents cases characterized by a smooth solution. Figure 5(right) will be discussed later on. The results show that there exists a critical curve $\epsilon_{c}\left(\phi_{0}\right)$ that can be traced in this plane, dividing tidal-bore and no-tidal-bore estuaries. This curve is the white dashed line traced in figure 5(left). Observing the shape of the isolines
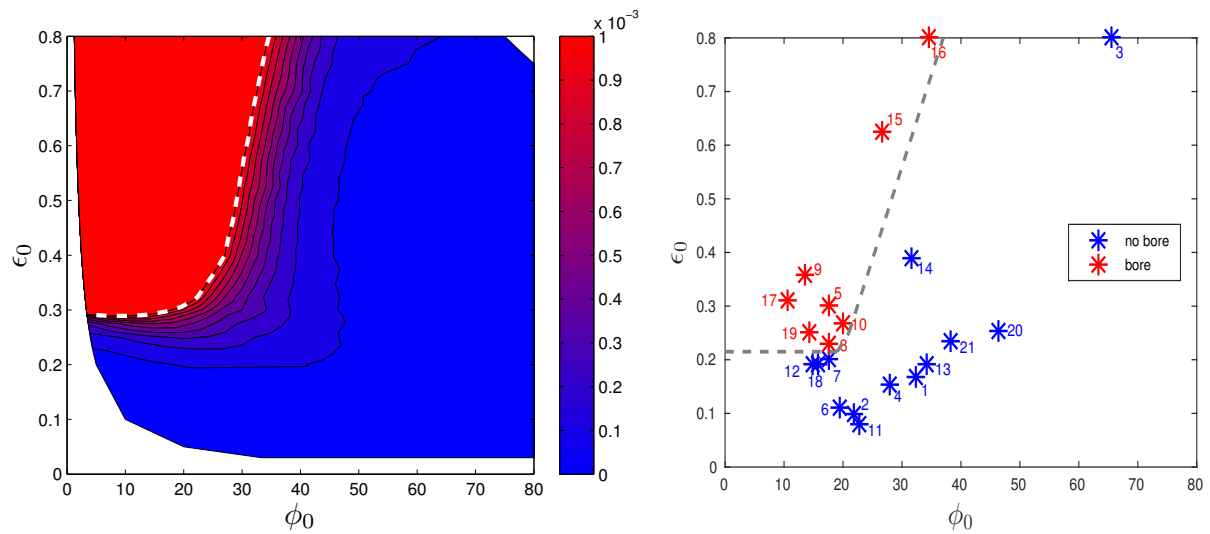

Figure 5: (Left): Isocurves of the quantity $A_{\max }$ in the plane of the parameters $\left(\phi_{0}, \epsilon_{0}\right)$, the white dashed line represents the $\epsilon_{c}\left(\phi_{0}\right)$ curve, namely the limit for tidal bore appearance following the criterion $A_{\max } \geq 10^{-3}$. (Right) : Projection of real alluvial estuaries data on the plane $\left(\phi_{0}, \epsilon_{0}\right)$; with a grey dashed line (- -) we have represented by hand the transition between the two regimes, inspired by the similar trend in the left picture. The database used to generate the picture can be found in table 2 of [13], for brevity and completeness we list here the name and number of the estuaries included in the picture: 1. Chao Phya; 2. Columbia; 3. Conwy; 4. Corantijin; 5. Daly; 6. Delaware; 7. Elbe; 8. Gironde; 9. Hooghly; 10. Humber; 11. Limpopo; 12. Loire; 13. Mae Klong; 14. Maputo; 15. Ord; 16. Pungue; 17. Qiantang; 18. Scheldt; 19. Severn; 20. Tha Chin; 21. Thames. Sources: 6] for estuaries 1, 4, 11, 13, $14,18,20$; 1 for $2,3,6,7,15,19,21$; 3 for $8,9,10,16,17$; 27 for 5 ; 28 for 12 .

in figure 5 (left), two different behaviors can be distinguished, depending on the values assumed by the friction parameter $\phi_{0}$.

The first region is characterized by values of the friction parameter in the 
range $1<\phi_{0} \leq \sim 20$. Here the mechanism of bore inception appears to be fully controlled by the nonlinear parameter $\epsilon_{0}$. Figure 6 shows a comparison between two numerical results computed using two different values of $\epsilon_{0}$, one just above the critical curve and one just below it $\left(\epsilon_{0}=0.3\right.$ and $\epsilon_{0}=0.225$ respectively), at a constant value of $\phi_{0}$ equal to 13.33. The comparison is made in terms of the free surface and velocity time series, respectively in figure 6(a) and 6(b), measured at the location $x=L_{c}$. In both figures, the signals associated to the case at higher $\epsilon_{0}$ are characterized by a greater distortion at the beginning of the flood phase, leading to bore formation according to our threshold. The two zooms, displayed in figures 6(c) and (d), allow to better appreciate the different time gradients exhibited by the water wave on the time scale of the tidal bore (around 20 minutes). It is also interesting to note that the free surface profile of figure 6(a) does not display a Burger's like shock, but rather a knee shape is observed. This is mainly due to the fact that the nonlinear effect of friction prevails on the advective one, remaining the dominant nonlinearity for the major part of the wave transformation. Figure 7 displays the relative importance of the various terms in the momentum equation during a tidal cycle in the previous case of bore development $\left(\epsilon_{0}=0.3\right.$ and $\left.\phi_{0}=13.33\right)$. In the figures, the time evolution of these quantities is represented at three equispaced locations along the channel, in particular at $x=\frac{1}{3} L_{c}, x=\frac{2}{3} L_{c}$ and $x=L_{c}$. It emerges that the advective term remains negligible in the momentum equation and the bore formation results from a balance between acceleration, friction and hydrostatic terms. Only in the presence of incipient bore, the relative importance of the advective term rises up overcoming the frictional nonlinearity. This behaviour can be also recovered when a dispersive bore development is simulated. For the previous case of undular bore formation of section 5 , the time variations of the relative magnitude for the momentum equation's terms are compared in figure 8. For sake of clarity, data are shown for the small time of the bore passage at two space locations: just before (left) and after (center and right) the appearence of the secondary waves. It can be seen that, as in the previous case of figure 7 , the advective term becomes the relevant nonlinear term on the 


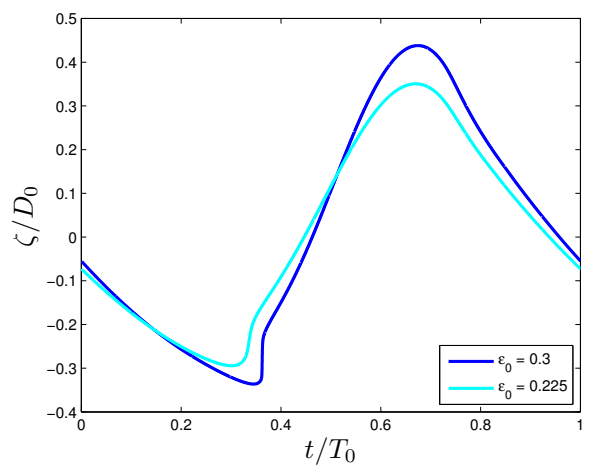

(a)

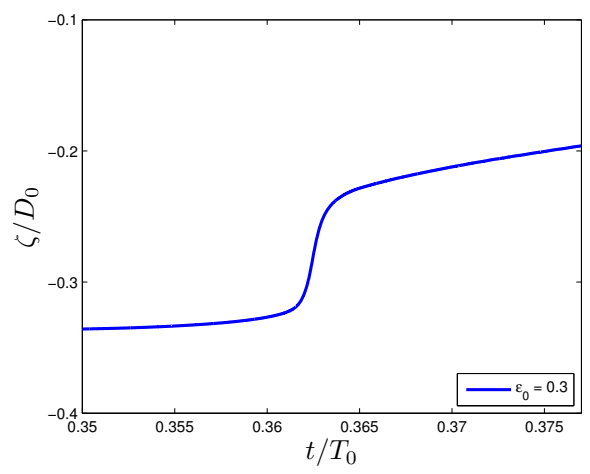

(c)

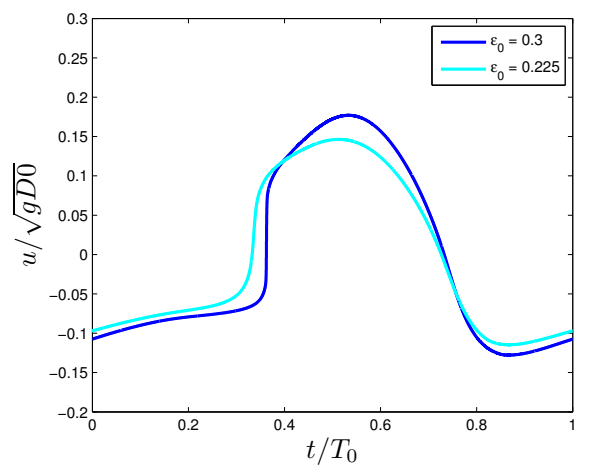

(b)

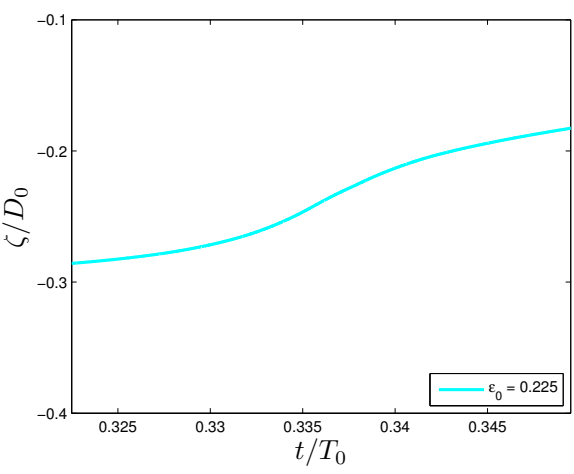

(d)

Figure 6: Time variation of the free surface elevation (a) and the velocity (b) signals measured at $x=L_{c}$ for a fixed value of $\phi_{0}=13.33$ and the two different values of $\epsilon_{0}=0.225(-)$ and $\epsilon_{0}=0.3(-)$. (c) and (d) are two zooms on the water wave profile at the beginning of the flood phase, on the time scale of the tidal bore (around 20 minutes).

bore location. However, in this case, its increase is partially limited by the rise of the dispersive term in a general context dominated by the balance between the inertial and the hydrostatic contributions.

For higher values of $\phi_{0}\left(\phi_{0} \geq \sim 20\right)$, the isolines of $A_{\max }$ spread out forming a wider transition region but, more important, display an almost vertical slope. This implies an increasing role of the friction parameter in the physical mechanism of bore formation. In figure 9, we show the solutions computed for 

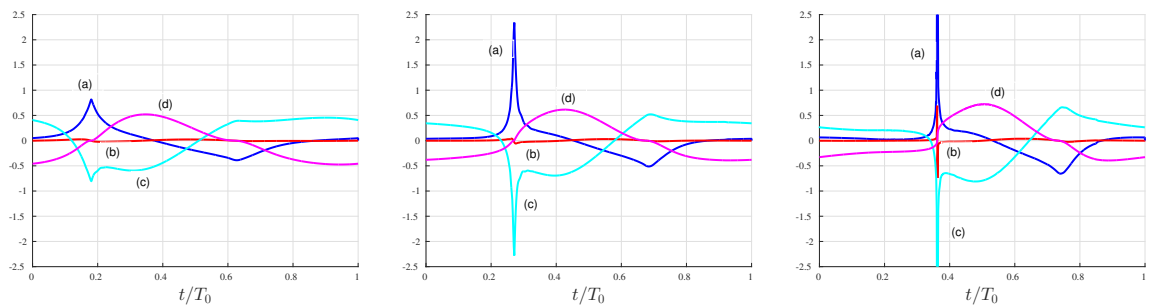

Figure 7: The order of magnitude of the several dimensionless terms in the momentum equation of system 17) without dispersion; three test sections are presented: at $x=\frac{1}{3} L_{c}$ (left), $x=\frac{2}{3} L_{c}$ (center) and $x=L_{c}$ (right): (a) $\frac{\partial \boldsymbol{u}}{\partial t}$, (b) $\frac{K}{\mathcal{L}} \epsilon_{0}(\boldsymbol{u} \cdot \nabla) \boldsymbol{u}$, (c) $\frac{1}{K \mathcal{L}} \delta_{0}^{2} \nabla \zeta$ and (d) $K \frac{\epsilon_{0} \phi_{0}}{\delta_{0}} \frac{\boldsymbol{u}|\boldsymbol{u}|}{D}$. Upper and lower peaks on the figure (right) reach values respectively of 30.65 and -28.61 .
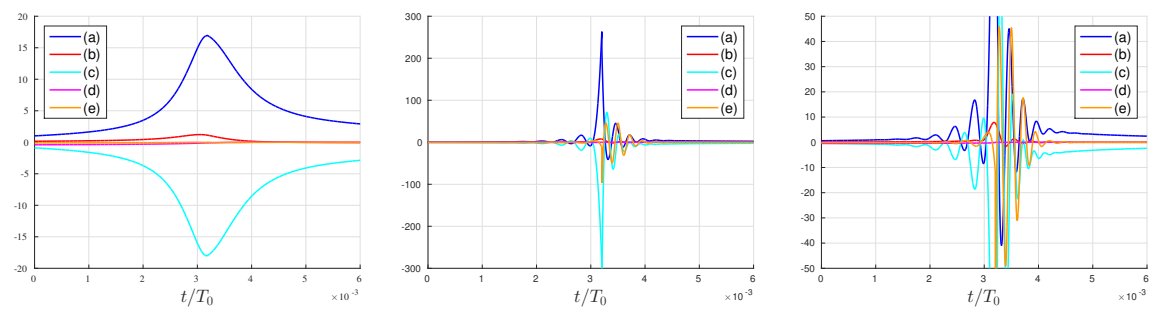

Figure 8: The order of magnitude of the several dimensionless terms in the momentum equation of system 17], including the dispersion; two test sections are presented: at $x=\frac{1.02}{3} L_{c}$ (left) and $x=\frac{1.12}{3} L_{c}$ (center), focusing on the time scale of the bore passage, while the third picture (right) represents, for clearness, a zoom of the central one: (a) $\frac{\partial \boldsymbol{u}}{\partial t}$, (b) $\frac{K}{\mathcal{L}} \epsilon_{0}(\boldsymbol{u} \cdot \nabla) \boldsymbol{u}$, (c) $\frac{1}{K \mathcal{L}} \delta_{0}^{2} \nabla \zeta$ and (d) $K \frac{\epsilon_{0} \phi_{0}}{\delta_{0}} \frac{\boldsymbol{u}|\boldsymbol{u}|}{D}$ and (e) $\mu^{2} \boldsymbol{\Psi}$.

two cases across the transition zone $\left(\phi_{0}=20\right.$ and $\phi_{0}=40$ respectively), keeping constant $\epsilon_{0}=0.4$. Once again, we look to the free surface and velocity time signals to compare the two results, figures 9(a) and 9(b) respectively. It clearly appears that an increase of the value of the friction parameter $\phi_{0}$ is directly associated to both potential and kinetic energy dissipation, leading to more damped profiles. This process decreases the local nonlinearity of the wave which, in turn, is smeared out, as one can see from the free surface zooms on the time scale of the bore (figures 9(c) and 9(d)). Moreover, figures 9(b) displays the particular tendency of the tidal current to become constant during the ebb tide for large values of the friction parameter (strongly dissipative estuaries), in 
agreement with previous studies (e.g. [1] and [3).

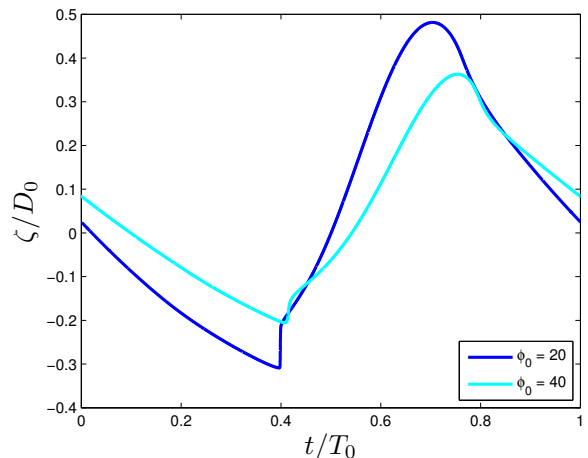

(a)

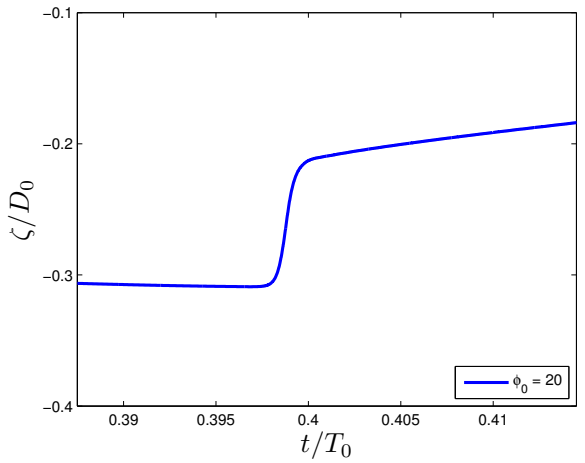

(c)

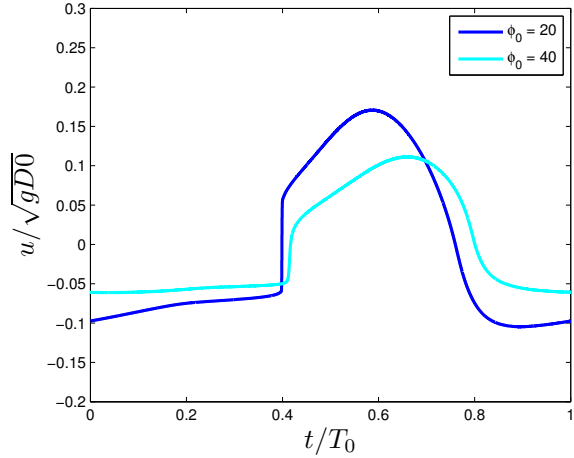

(b)

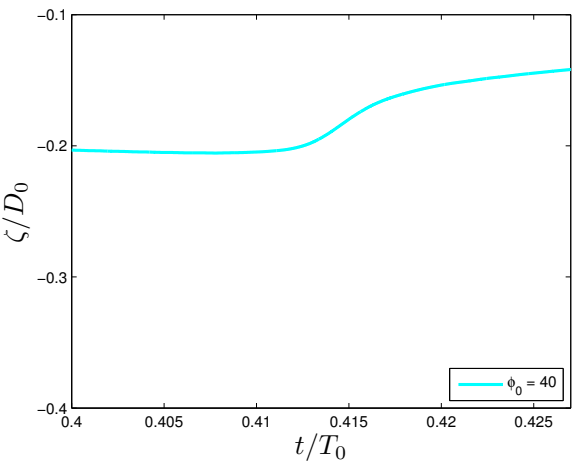

(d)

Figure 9: Time variation of the free surface elevation (a) and the velocity (b) signals measured at $x=L_{c}$ for a fixed value of $\epsilon_{0}=0.4$ and the two different values of $\phi_{0}=20(-)$ and $\phi_{0}=40$ $(-)$. (c) and (d) are two zooms on the water wave profile at the beginning of the flood phase, on the time scale of the tidal bore (around 20 minutes).

We can schematically summarize that the nonlinear parameter $\epsilon_{0}$ mainly relates to the distortion mechanism which leads to bore formation, while the friction parameter $\phi_{0}$ mainly relates the dissipation of the tidal wave, unfavorable to bore generation.

The separation between estuaries displaying or not a tidal bore, which emerges from our numerical results, is in qualitative agreement with real-estuary observations analyzed in [13. A list of tidal and geometrical properties for 21 conver- 
gent alluvial estuaries ( 8 of them displaying a bore and 13 not) has been used in [13] to produce figure 5(right), reported here for sake of clarity. In the picture, a grey dashed line dividing tidal-bore and no-tidal-bore estuaries has been traced by hand, inspired by the trend of the critical curve emerged in figure 5 (left). We can notice that the numerical critical curve $\epsilon_{c}\left(\phi_{0}\right)$ and the data-based one only slightly differ considering the several simplifying modeling assumptions. In particular, in real alluvial estuaries the general decrease of depth landward is favorable to bore inception and this can cause the critical data-based curve (figure $2 \mathrm{~b}$ of [13]) to be located slightly below with respect to the modeling one on figure 5 (left).

From the observations made in the previous paragraph, a close relation between bore formation and tidal damping emerges. A standard parameter used in the literature [29, 30] to measure the amplification/damping of the tidal wave during its propagation along the estuary is the rate of change of the tidal range Tr, defined in accordance to reference [30] as:

$$
\delta_{\operatorname{Tr}}=\frac{1}{\operatorname{Tr}} \frac{d \operatorname{Tr}}{d x} .
$$

In the present work we integrate equation (8), from the estuary mouth to $x=L_{c}$ (end of our region of study), and we compute, for each simulation performed, the quantity:

$$
\Delta_{\operatorname{Tr}}=\frac{\operatorname{Tr}\left(L_{c}\right)-\operatorname{Tr}(0)}{\operatorname{Tr}(0)}
$$

using the tidal range at the estuary mouth $\operatorname{Tr}(0)$ as a scaling factor.

Figure 10(left) shows, on the same plane $\left(\phi_{0}, \epsilon_{0}\right)$ of figure 5 the contour lines of the computed quantity $\Delta_{\mathrm{Tr}}$, obtained by linearly interpolating the values of each simulation. The black dashed line is the contour line for $\Delta_{\mathrm{Tr}}=0$, namely the marginal curve for tidal range amplification, where all the estuaries with unamplified and undamped wave lie. It represents an ideal situation for which the amplification effect associated to funneling is exactly balanced by friction. The marginal curve divides the plane into two regions; estuaries located below are characterized by a tidal range amplification while estuaries located above are affected by damping. A simple analytical model, derived by Savenije et al. 
[29] in the linearized case, allows to explicitly compute the damping factor of an estuary as a function of the three external independent parameters of the estuarine dynamics. According to this model, Toffolon et al. [2] found that synchronous estuaries $\left(\delta_{\operatorname{Tr}}=0\right)$ lie on a curve, which can be recast in terms of our parameters as:

$$
\epsilon_{0}=\frac{\delta_{0}\left(\delta_{0}^{2}+1\right)}{\phi_{0}} .
$$

Using the value of $\delta_{0}=2$ in 10 , we get the red curve plotted in figure 10 (left). A good agreement is observed between the marginal curve obtained through numerical simulations (black dashed line) and the one of the analytical model of Savenije (red line), especially for low values of $\epsilon_{0}$, namely the linear regime.

From figure 10 (left) it is interesting to note that $\epsilon_{0}$ plays a fundamental role also in the damping/amplification process. If we fix the physical and geometrical properties $L_{b}, C_{f 0}, D_{0}$, this corresponds to a specific estuary configuration with $\phi_{0}$ constant in addition to $\delta_{0}=2$. In this context, the variations of $\epsilon_{0}$ can be considered as associated with neap-spring tide cycles. We can, thus, conclude that strong tides lead to weaker tidal wave amplification values and, for particularly strong tides, the wave most likely will be damped. This result is in qualitative agreement with the physical observation made in [3] for the Garonne river. Their measurements showed that, for such estuary always characterized by tidal wave amplification, minor amplifications were related to higher values of $\epsilon_{0}$, observed during spring tides.

The superimposition of the computed marginal curve on the contour lines of the quantity $A_{\max }$, in figure 10 (right), shows that a large part of the red region in the figure, lies in the part of the plane $\left(\phi_{0}, \epsilon_{0}\right)$ characterized by damping of the tidal range. This means that, despite a reduction of the local nonlinearity of the wave, this remains high enough to develop distorted profiles and to produce bores. We can conclude that, contrary to what is generally accepted, tidal range amplification along the estuary is not a necessary condition for tidal bore formation. 

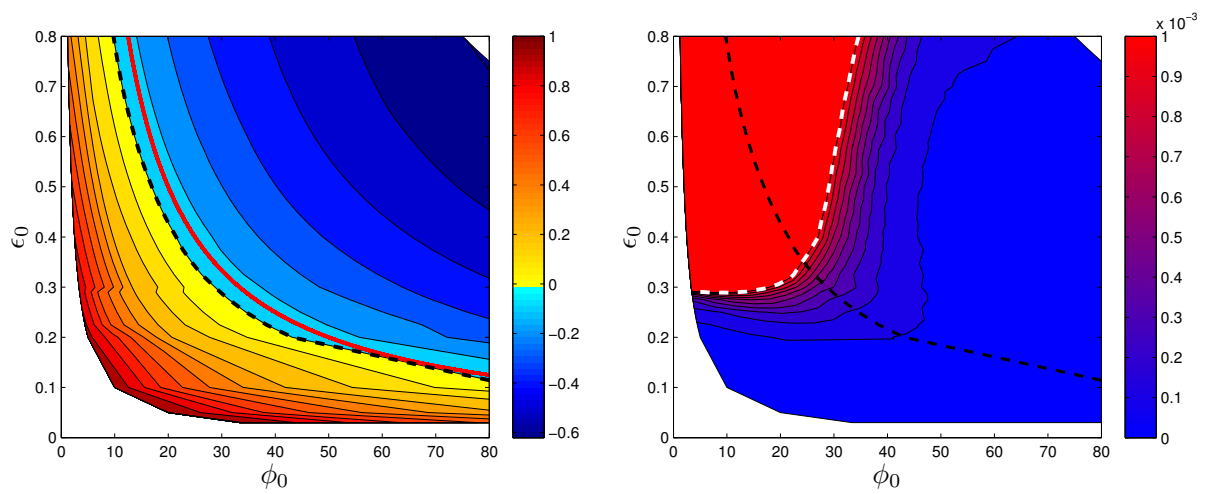

Figure 10: (Left): contour lines of the quantity $\Delta_{\operatorname{Tr}}$ on the plane $\left(\phi_{0}, \epsilon_{0}\right)$; the black dashed line (- -) represents the marginal curve resulting from the computations, while the continuous red one $(-)$ is the analytical marginal curve of the Savenije model [30. Hot colors cover the region of amplification of the tidal wave during propagation; cold colors represent damping. (Right): the computed marginal curve (- -) is superimposed on the $A_{\max }$ contour lines.

\section{Influence of river discharge}

In the previous sections, we have analyzed tidal wave transformation and tidal bore occurrence in a simplified context in which freshwater river discharge was neglected. However, it is well known that tide in estuaries may be significantly affected by the rate of discharge [16, 5]. The effects of river discharge become much more important moving landward from the mouth of the estuary and can influence for bore formation. The present section will provide a qualitative estimation on the effects of discharge, leaving a full quantitative study for future works.

In the experimental campaigns on the Garonne river, Bonneton et al. 3] observed that small river discharges $Q$ were favorable to tidal range amplification and bore occurrence, while significant freshwater discharges offsets the amplification mechanism related to estuary convergence. Horrevoets et al. [16] described, with an analytical model, that the influence of river discharge on tidal damping takes place mainly through friction. Generally speaking, the tidally averaged free surface elevation along the estuary does not coincide with the mean sea level, due to the nonlinear frictional effect on the averaged water level 
$\bar{D}(x)$. In practice there is a monotone increase of $\bar{D}(x)$ landward, proportional to the river discharge $Q$, see [31, 5]. Moreover, a damping of the local tidal range $\operatorname{Tr}(x)$ has been pointed out by Bonneton et al. [3], hence the local nonlinear parameter $\epsilon(x)=\operatorname{Tr}(x) /(2 \bar{D}(x))$ is a decreasing function of $Q$. Due to this damping effect, tidal bores are rarely observed for strong freshwater river discharges.

For a fixed estuary (fixed $\delta_{0}$ and $\phi_{0}$ ), the dimensionless parameters governing the flow dynamics are the amplitude of tidal forcing $\epsilon_{0}$ and the dimensionless intensity of river discharge $Q_{0}$. The goal of the present section is to explore the space of parameters $\left(\epsilon_{0}, Q_{0}\right)$ in order to find, for an estuary characterized by $\delta_{0}=2$ and $\phi_{0}=18$ (values closed to the ones of the Garonne river), a critical curve $\epsilon_{c}\left(Q_{0}\right)$ for tidal bore development, following the criterion $A_{\max } \geq 10^{-3}$. In order to perform our investigation, we have to express $Q_{0}$ as a function of the external variables of the problem. The scaling analysis proposed in [13] leads to the definition of a parameter $K=\frac{U_{0} D_{0}}{A_{0} \omega_{0} L_{b}}$ (see also appendix A for details), that has been measured to be $K \sim 1$ in convergent alluvial estuaries [3]. A characteristic velocity scale $U_{0}=\epsilon_{0} \omega_{0} L_{b}$ can, thus, be derived. Being $B_{0}$ the width at the estuary mouth (figure 1), we finally define:

$$
Q_{0}=\frac{Q}{A_{0} B_{0} L_{b} \omega_{0}} .
$$

The river discharge is introduced by the boundary condition already described in section 3 through the incoming Riemann invariant from far on the right. Figures 11 shows, for the particular estuary considered, the effect of an increasing river flow in terms of normalized free surface elevation and velocity signals at the position $x=L_{c}$ along the channel. The simulations were performed using $\epsilon_{0}=0.32$ and a range of values $Q_{0} \in\left[0,4.16 \times 10^{-3}\right]$, obtained by scaling the typical values of the Garonne river through relation (11). In particular, the values $Q_{0}=4.16 \times 10^{-4}$ and $Q_{0}=4.16 \times 10^{-3}$ correspond to the low and high characteristic fresh water discharges measured in the Garonne (respectively $Q=150\left[\mathrm{~m}^{3} / \mathrm{s}\right]$ and $\left.Q=1500\left[\mathrm{~m}^{3} / \mathrm{s}\right]\right)$. In figure 11 (a), we observe that the dimensionless mean water depth $\bar{D}\left(L_{c}\right) / D_{0}$ increases with $Q_{0}$, from 1.033 with 
$Q_{0}=0$ to 1.219 with $Q_{0}=4.16 \times 10^{-3}$. We can also measure the damping effect of freshwater river discharge on the tidal range; the dimensionless value $\Delta_{T r}\left(L_{c}\right)$ (as defined in (8)) goes from 0.156 with $Q_{0}=0$ (amplified case) to -0.041 with $Q_{0}=4.16 \times 10^{-3}$ (damped case). An important vertical shift of the velocity curve, in agreement with field observations [16], can be observed in figure 11 (b), moving towards the condition of unidirectional flow. Moreover, it can be noticed that this result confirms the theoretical predictions set by Horrevoets et al. [16] concerning the evolution of the phase lag between high water and high water slack (and at the same time between low water and low water slack). Note that all the effects described are small below the value of $Q_{0}=4.16 \times 10^{-4}$ and the discharge does not affect the topology of the tidal wave for the river flow values typically observed in the Garonne river at the end of the summer season. Thus, the parametric analysis performed in section 2 by neglecting $Q_{0}$, can be considered valid in this range of small $Q_{0}$.

In figure 11 (c), the zoom on the time scale of the bore displays the tendency of the free surface profile to become much smoother as the value of river discharge increases. In order to explore better this point, we have performed 47 simulations for different combinations of tidal amplitude and river discharge $\left(\epsilon_{0}\right.$, $\left.Q_{0}\right)$. The values of $A_{\max }$, obtained for all the simulations, have been plotted in figure 12 . Note that we chose to represent in the $y$-axis of the figure the product $Q_{0} \epsilon_{0}$, rather than simply $Q_{0}$, in order to remove the dependence of $Q_{0}$ from $A_{0}$ 11. The figure shows that, in the presence of weak river discharges, estuarine dynamics is not influenced by $Q_{0}$ and, consequently, the effects of discharge can be considered negligible in the bore formation process. A qualitative critical curve $\epsilon_{c}\left(Q_{0}\right)$ has been traced by hand (grey dashed line in figure 12 according to the few computations performed and following the criterion $A_{\max } \geq 10^{-3}$. This trend is in qualitative agreement with experimental data for the Garonne river, presented in 13 . 


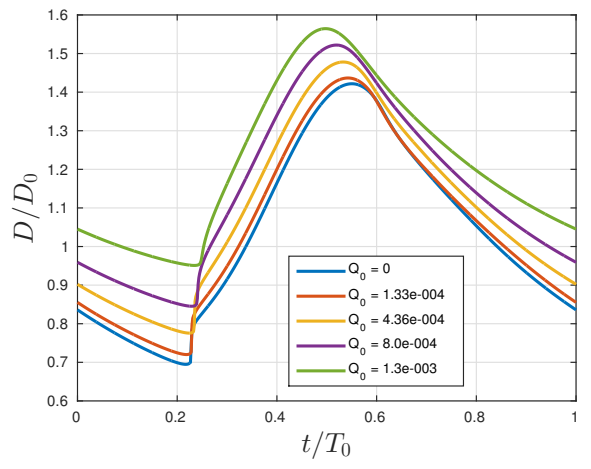

(a)

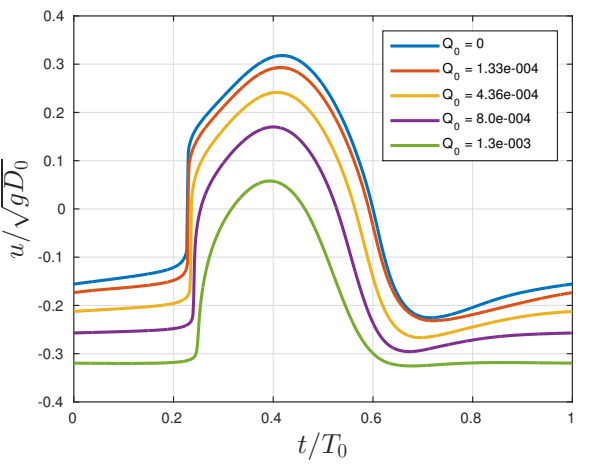

(b)

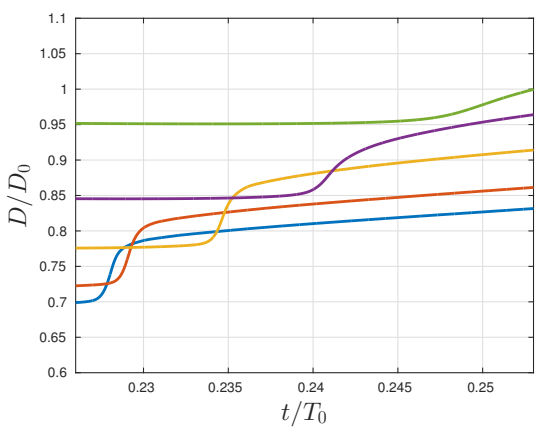

(c)

Figure 11: Time variation of the water depth (a) and velocity (b) signals measured at $x=3 L_{b}$ for an ideal estuary characterized by $\epsilon_{0}=0.32, \phi_{0}=18$ and increasing values of freshwater discharge from $Q_{0}=0$ to $Q_{0}=4.16 \times 10^{-3}$ (in particular the values have been chosen considering the typical range of values displayed by the Garonne river and measured by Bonneton et al. [3]). Figure (c) represents a zoom on (a) in the time scale of the bore (around $20 \mathrm{~min}$ ).

\section{Conclusions}

The two-dimensional Serre-Green-Naghdi system of equations has been used in order to simulate the inception of tidal bores in convergent alluvial estuaries of idealized geometry. Exploiting the dispersive properties of the model, we were, thus, the first to reproduce the formation of an undular tidal bore inside a channel, to the authors' knowledge. The results obtained have illustrated that dispersion does not impact the large scale of propagation of the tide, as it comes 


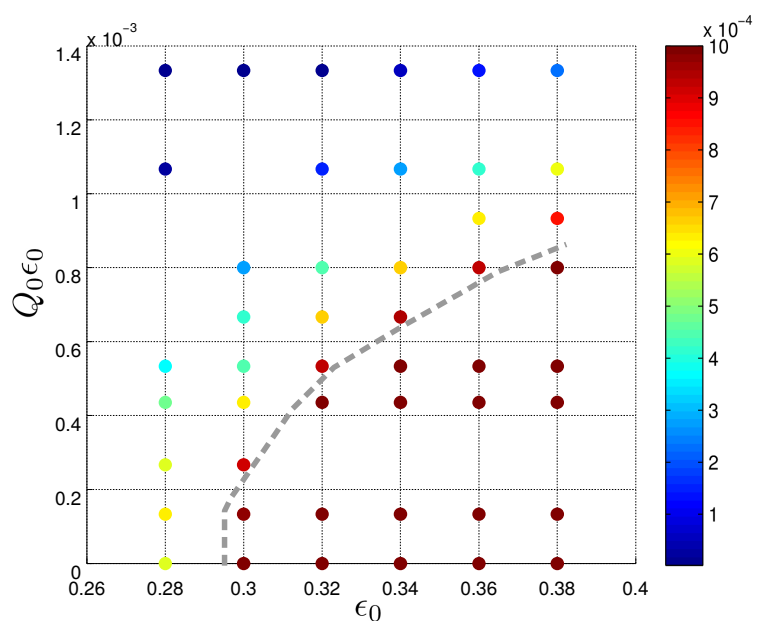

Figure 12: Circles represent computations performed for a fixed estuary $\left(\delta_{0}=2\right.$ and $\left.\phi_{0}=18\right)$ varying the values of the tidal forcing amplitude $A_{0}$ and river discharge $Q_{0}$; colors represent the intensity of $A_{\max }$ for each computation; with a grey dashed line we have represented by hand the $\epsilon_{c}\left(Q_{0}\right)$ curve, namely the limit for tidal bore appearance following the criterion $A_{\max } \geq 10^{-3}$

also from the scaling analysis of the equations proposed in appendix A, and affects the solution only in the proximity of bore formation. For these reasons, an accurate description of the free surface profile at large scale can be obtained via the simpler NLSW system. Under the hypotheses of constant bathymetry and negligible river discharge, we have thus employed the NLSW equations to numerically investigate the bore occurrence in convergent alluvial estuaries of idealized geometry.

The scaling of the equations shows that estuarine dynamics is fully controlled by three dimensionless parameters entirely dependent on the estuary geometrical properties and tidal forcing: the nonlinearity $\epsilon_{0}$, the convergence parameter $\delta_{0}$ and the friction parameter $\phi_{0}$, defined by $(3)$. Taking a constant value of $\delta_{0}=2$, we have numerically explored the space $\left(\phi_{0}, \epsilon_{0}\right)$. By means of a bore detection criterion, we have traced the critical line $\epsilon_{c}\left(\phi_{0}\right)$ dividing estuaries into displaying or not a tidal bore. This curve is in good agreement with real estuaries data, despite the several assumptions made, and provides the necessary 
The critical curve intersects the computed marginal curve of amplification, di-

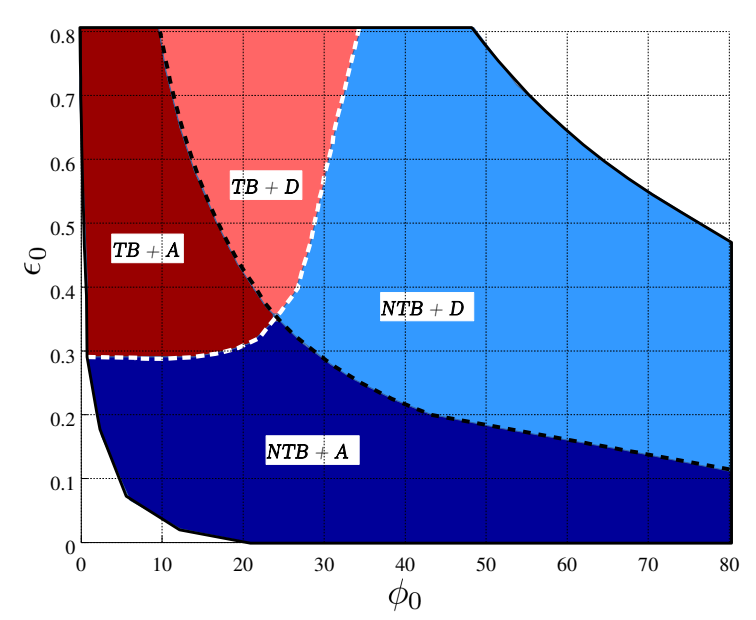

Figure 13: The computed critical line for bore formation (white dashed line) and the computed marginal curve of amplification (black dashed line) divide the $\left(\phi_{0}, \epsilon_{0}\right)$ plane into four main areas. In the picture, TB stands for tidal-bore estuaries, while NTB stands for no tidal-bore estuaries; A indicates amplification of the tidal wave along the estuary, D damping.

conditions for tidal bore formation. These conditions are a result of a complex equilibrium between nonlinear distortion and tidal range damping/amplification processes both driven by the dissipation parameter $D_{i}^{*} \propto \epsilon_{0} \phi_{0}$ multiplying the friction, the dominant nonlinear term for this class of estuaries. The particular shape of $\epsilon_{c}\left(\phi_{0}\right)$ shows that, for low values of $\phi_{0}$ (indicatively $1<\phi_{0} \leq \sim 20$ ), bore formation depends almost exclusively on the nonlinear parameter $\epsilon_{0}$, while being, instead, mainly disciplined by the dissipation, related to the value of $\phi_{0}$, for $\phi_{0} \geq \sim 20$

\footnotetext{
viding the plane into four main areas. Estuaries will thus experience tidal range amplification or damping, tidal bore formation or not, depending on which region in the plane they belong to (see figure 13). The existence of a sector characterized by tidal bore generation and tidal range damping shows that tidal range amplification along the estuary is not a necessary condition for tidal bore occurrence, as it is instead commonly assumed in the literature 32]. This result is also in agreement with field observations analyzed in [13.
} 
Finally, we have studied the effect of river discharge for estuaries characterized by $\delta_{0}=2$ and $\phi_{0}=18$ (which are close to the values displayed by the Gironde/Garonne estuary). We have shown that for low $Q_{0}$ (i.e. $Q_{0}<$ $4.16 \times 10^{-3}$ ), corresponding to the dry season, the effect of river discharge on tidal wave dynamics and bore formation can be neglected.

The above findings are based on several simplifying assumptions, that have allowed a clear understanding of the bore inception mechanism. Other effects influencing the spatial location of bore development have been so far ignored and may arise due to the variable bathymetry, river banks and meanders. The consideration of such effects will require significant attention, but will provide a more thorough comprehension when approaching the analysis of real natural estuaries.

\section{Appendix A: Scaling of the SGN equations}

In this section we report some details concerning the application of the scaling proposed in [13, to the SGN system of equations (5). The following scaling of the physical variables is thus applied:

$$
\begin{aligned}
x=L_{0} x^{\prime} ; \quad y & =L_{0} y^{\prime} ; \quad t=\frac{t^{\prime}}{\omega_{0}} ; \quad D=D_{0} D^{\prime} ; \\
\zeta & =A_{0} \zeta^{\prime} ; \quad \boldsymbol{u}=U_{0} \boldsymbol{u}^{\prime} .
\end{aligned}
$$

The governing equations (5a) and (5b), written in dimensionless form, will thus read (dropping the primes for sake of clarity):

$$
\begin{aligned}
\frac{\partial \zeta}{\partial t}+\frac{K}{\mathcal{L}}\left(\epsilon_{0} \boldsymbol{u} \nabla \zeta+D \nabla \cdot \boldsymbol{u}\right) & =0 \\
\frac{\partial u}{\partial t}+\frac{K}{\mathcal{L}} \epsilon_{0}(\boldsymbol{u} \cdot \nabla) \boldsymbol{u}+\frac{1}{K \mathcal{L}} \delta_{0}^{2} \nabla \zeta+K \frac{\epsilon_{0} \phi_{0}}{\delta_{0}} \frac{\boldsymbol{u}|\boldsymbol{u}|}{D} & =\boldsymbol{\psi} .
\end{aligned}
$$

where $\mathcal{L}=\frac{L_{0}}{L_{b}}$ and $K=\frac{U_{0} D_{0}}{A_{0} \omega_{0} L_{b}}$ are functions of the length and velocity scales of the phenomenon [13] and the scale factor $\frac{2 \pi U_{0}}{T_{0}}$ has been used for the nonhydrostatic term $\boldsymbol{\psi}$. 
Considering a flat bathymetry, the linear operators $\mathrm{T}(\boldsymbol{w})$ and $\mathrm{Q}(\boldsymbol{w})$, applied to a generic vector $\boldsymbol{w}$, are:

$$
\begin{aligned}
& \mathrm{T}(\boldsymbol{w})=-\frac{1}{3 D} \nabla\left(D^{3} \nabla \cdot \boldsymbol{w}\right), \\
& \mathrm{Q}(\boldsymbol{w})=\frac{2}{3 D} \nabla\left[D^{3}\left(\nabla \boldsymbol{w}_{1} \cdot \nabla^{\perp} \boldsymbol{w}_{2}+(\nabla \cdot \boldsymbol{w})^{2}\right)\right] ;
\end{aligned}
$$

in which $\boldsymbol{w}_{1}$ and $\boldsymbol{w}_{2}$ indicates respectively the first ans second component of the vector $\boldsymbol{w}$ and $\nabla^{\perp}$ stays for the normal gradient operator. Applying the scaling (12) to the two expressions above and introducing the dimensionless dispersion parameter $\mu=\frac{D_{0}}{L_{0}}$, we can state that $\mathrm{T}(\boldsymbol{w})=\mu^{2} \mathrm{~T}^{\prime}(\boldsymbol{w})$ and $\mathrm{Q}(\boldsymbol{w})=\frac{\mu^{2}}{L_{0}} \mathrm{Q}^{\prime}(\boldsymbol{w})$. Equation (5c), written in terms of dimensionless variables, then becomes (still primes are dropped for clarity):

$$
\left(\mathrm{I}+\mu^{2} \mathrm{~T}\right) \boldsymbol{\psi}=\mu^{2} \mathrm{~T}\left(\frac{1}{K \mathcal{L}} \delta_{0}^{2} \nabla \zeta-K \frac{\epsilon_{0} \phi_{0}}{\delta_{0}} \frac{\boldsymbol{u}|\boldsymbol{u}|}{D}\right)-\mu^{2} \mathrm{Q}\left(\frac{K}{\mathcal{L}} \epsilon_{0} \boldsymbol{u}\right)
$$

Equation (15) can be rearranged as follows:

$$
\boldsymbol{\psi}=\mu^{2}\left(\mathrm{I}+\mu^{2} \mathrm{~T}\right)^{-1}\left[\mathrm{~T}\left(\frac{1}{K \mathcal{L}} \delta_{0}^{2} \nabla \zeta-K \frac{\epsilon_{0} \phi_{0}}{\delta_{0}} \frac{\boldsymbol{u}|\boldsymbol{u}|}{D}\right)-\mathrm{Q}\left(\frac{K}{\mathcal{L}} \epsilon_{0} \boldsymbol{u}\right)\right],
$$

showing that $\boldsymbol{\psi}=\mu^{2} \boldsymbol{\Psi}$.

The final form of scaled SGN system will thus read:

$$
\begin{gathered}
\frac{\partial \zeta}{\partial t}+\frac{K}{\mathcal{L}}\left(\epsilon_{0} \boldsymbol{u} \nabla \zeta+D \nabla \cdot \boldsymbol{u}\right)=0 \\
\frac{\partial u}{\partial t}+\frac{K}{\mathcal{L}} \epsilon_{0}(\boldsymbol{u} \cdot \nabla) \boldsymbol{u}+\frac{1}{K \mathcal{L}} \delta_{0}^{2} \nabla \zeta+K \frac{\epsilon_{0} \phi_{0}}{\delta_{0}} \frac{\boldsymbol{u}|\boldsymbol{u}|}{D}=\mu^{2} \boldsymbol{\Psi} \\
\boldsymbol{\Psi}=\left(\mathrm{I}+\mu^{2} \mathrm{~T}\right)^{-1}\left[\mathrm{~T}\left(\frac{1}{K \mathcal{L}} \delta_{0}^{2} \nabla \zeta-K \frac{\epsilon_{0} \phi_{0}}{\delta_{0}} \frac{\boldsymbol{u}|\boldsymbol{u}|}{D}\right)-\mathrm{Q}\left(\frac{K}{\mathcal{L}} \epsilon_{0} \boldsymbol{u}\right)\right]
\end{gathered}
$$

\section{Appendix B: Landward boundary conditions}

$$
\text { Imposing correctly the landward boundary condition (b.c.) is not a trivial }
$$
operation. Up to the authors knowledge, there is not an efficient and systematic method to impose an outflow conditions in the presence of friction and convergence. In this appendix a sensitivity analysis has been performed in order to quantify the influence of the inexact b.c. that has been implemented for this 
study ( $c f$. section 3.3). Two tests have been conducted: one for a low and the other for a high value of the nonlinear parameter $\epsilon_{0}$, respectively $\epsilon_{0}=0.1$ and $\epsilon_{0}=0.7$. We compare two solutions: one computed by setting the outflow b.c. at the location $x=5 L_{b}$, the other, considered as a reference, derived by imposing a wall b.c. at the further distance $x=8 L_{b}$, where the tidal wave is assumed to be completely dissipated. Figure 14 shows the time evolution profiles of the non-dimensional free surface and velocity at different positions along the channel. In order to measure the deviation from the reference solution we use the $L_{2}$-norm $\frac{\left\|\zeta-\zeta_{\text {ref }}\right\|_{L_{2}}}{\left\|\zeta_{\text {ref }}\right\|_{L_{2}}}$. At the station $x=3 L_{b}$, the percentage values of the deviation are $3.3 \%$ for $\zeta$ and $3.98 \%$ for $u$ in the case with $\epsilon_{0}=0.1$, while being respectively $5.27 \%$ and $7.67 \%$ in the case with $\epsilon_{0}=0.7$.

Toffolon [21] revealed the difficulties of imposing such conditions. He considered two limit cases: the reflecting barrier and the transparent condition. On the former, a wall boundary condition was imposed at the end of the channel, which determined a complete reflection of the wave. The latter condition, instead, referred to a situation where the tidal wave exited from the computational domain without being deformed or reflected (transparent b.c.). This condition was implemented by replacing, on the last cells of the domain, mass and momentum conservation laws with a scalar advection equation for each conservative variable, characterized by an advection speed equal to the outgoing eigenvalue. Even though, the need of considering a longer estuary in order to vanish the influence of the boundary on the solution, remained.

Figure 15 shows the comparison with the solution performed by implementing the transparent boundary condition used in [21. The percentage deviations from the reference are of the same order of magnitude as those recovered using our approach and, most importantly, the two approaches provide identical topologies for both the water depth and the velocity. The results of our study are thus independent from the choice of the method used. 

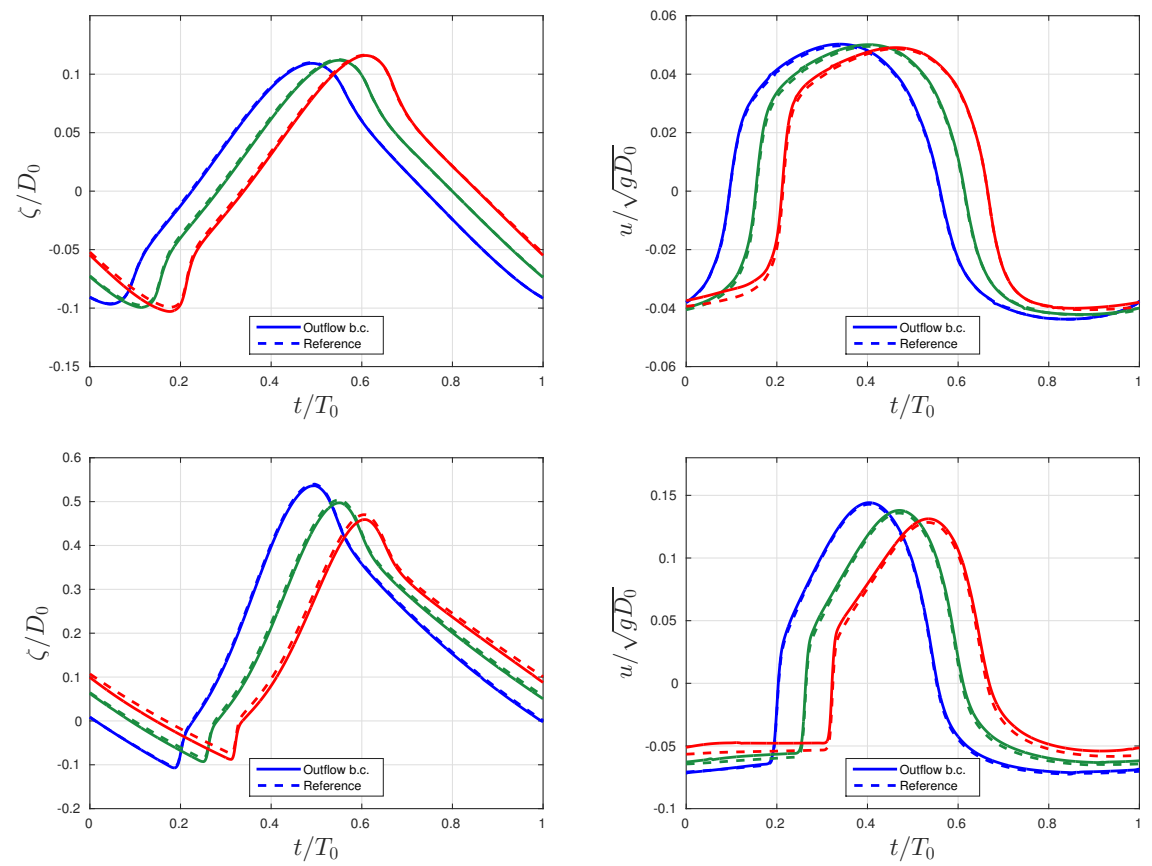

Figure 14: Time evolution profiles of the non-dimensional free surface (left) and velocity (right) measured at $x=2 L_{b}(-), x=2.5 L_{b}(-)$ and $x=3 L_{b}(-)$ for the two test cases performed with $\epsilon_{0}=0.1$ (top) and $\epsilon_{0}=0.7$ (bottom). In the two computations $\phi_{0}$ has been set constant and equals to $\phi_{0}=35$. Continuous lines represent the results obtained by using absorbing landward b.c. by means of the homogeneous NLSW invariants at $x=5 L_{b}$; while dashed lines were obtained by imposing wall b.c. at $x=8 L_{b}$.

\section{Acknowledgments}

Experiments presented in this paper were carried out using the PlaFRIM experimental platform, developed under the INRIA PlaFRIM development action with support from LABRI and IMB and other entities: Conseil Régional d'Aquitaine, FeDER, Université de Bordeaux and CNRS. This work was partially funded by the TANDEM contract, reference ANR-11-RSNR-0023-01 of the French Programme Investissements d'Avenir. 

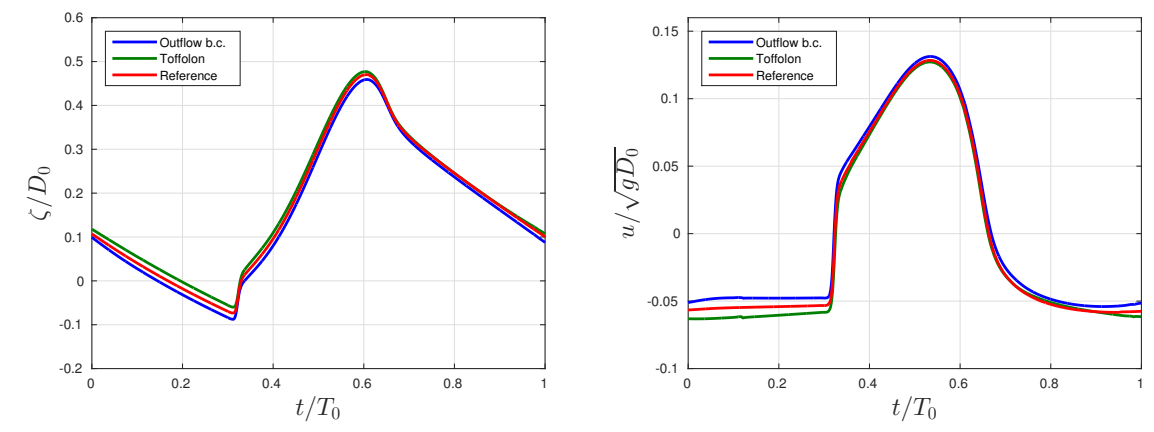

Figure 15: Time evolution profiles of the non-dimensional free surface (left) and velocity (right) measured at $x=3 L_{b}$, obtained by setting the absorbing homogeneous b.c. $(-)$ and the transparent b.c. of 21] (-). The red curve (-) represents the reference solution computed by imposing wall b.c. on a longer domain of $L=8 L_{b}\left(\epsilon_{0}=0.7\right.$ and $\left.\phi_{0}=35\right)$.

\section{References}

[1] S. Lanzoni, G. Seminara, On tide propagation in convergent estuaries, J. Geophys. Res. 103 (1998) 30793-30812.

[2] M. Toffolon, G. Vignoli, M. Tubino, Relevant parameters and finite amplitude effects in estuarine hydrodynamics, J. Geophys. Res. 111 (2006) $1-17$.

[3] P. Bonneton, N. Bonneton, J. P. Parisot, B. Castelle, Tidal bore dynamics in funnel-shaped estuaries, J. Geophys. Res. 120 (2015) DOI: 10.1002/2014JC010267.

[4] A. Munchow, R. W. Garvin, Nonlinear barotropic tides and bores in estuaries, Tellus 43 (1991) 246-256.

[5] H. Cai, H. H. G. Savenije, M. Toffolon, Linking the river to the estuary: influence of river discharge on tidal damping, Hydrol. Earth Syst. Sci. 18 (2014) 287-304.

[6] H. H. G. Savenije, Salinity and tides in alluvial estuaries, 2nd Edition, 2012. 
[7] D. H. Peregrine, Calculations of the development of an undular bore, J.Fluid Mech. 25 (1966) 321-330.

[8] P. A. Madsen, H. J. Simonsen, C. H. Pan, Numerical simulation of tidal bores and hydraulic jumps, Coast. Eng. 52 (2005) 409-433.

[9] C. H. Pan, H. Lu, 2d numerical simulation of tidal bore on Qiantang river using KFVS scheme, Vol. 32, 2010.

[10] A. E. Green, P. M. Naghdi, A derivation of equations for wave propagation in water of variable depth, J.Fluid Mech. 78 (1976) 237-246.

[11] M. Tissier, P. Bonneton, F. Marche, F. Chazel, D. Lannes, Nearshore dynamics of tsunami-like undular bores using a fully nonlinear Boussinesq model, J. Coast. Res. 64 (2011) 603-607.

[12] G. A. El, R. H. Grimshaw, N. F. Smyth, Unsteady undular bores in fully nonlinear shallow water theory, Phys. Fluids 18(2).

[13] P. Bonneton, A. G. Filippini, L. Arpaia, N. Bonneton, M. Ricchiuto, Conditions for tidal bore formation in convergent alluvial estuaries, Estuarine, Coastal and Shelf Science, doi:10.1016/j.ecss.2016.01.019.

[14] G. Davies, C. D. Woodroffe, Tidal estuary width convergence: Theory and form in North Australian estuaries, Earth Surf. Process. Landforms 35 (2010) 737749.doi: 10.1002/esp.1864.

[15] C. T. Friedrichs, D. G. Aubrey, Tidal propagation in strongly convergent channels, J. Geophys. Res. 99(C2) (1994) 33213336.doi: 10.1029/93JC03219.

[16] A. C. Horrevoets, H. H. G. Savenije, J. N. Schuurman, S. Graas, The influence of river discharge on tidal damping in alluvial estuaries, J. Hydrol. 294 (2004) 213-228.

[17] H. H. G. Savenije, M. Toffolon, J. Haas, M. Veling, Analytical description of tidal dynamics in convergent estuaries, J. Geophys. Res. 113 (2008) 1-18. 
[18] P. Winckler, P. L. Liu, Long waves in a straight channel with non-uniform cross-section, J.Fluid Mech. 770 (2015) 156-188.

[19] P. Bonneton, F. Chazel, D. Lannes, F. Marche, M. Tissier, A splitting approach for the fully nonlinear and weakly dispersive Green-Naghdi model, J.Comp.Phys. 230(4) (2011) 1479-1498.

[20] A. G. Filippini, M. Kazolea, M. Ricchiuto, A flexible genuinely nonlinear approach for nonlinear wave propagation, breaking and run-up, J.Comp.Phys. 310 (2016) 381417.

[21] M. Toffolon, Hydrodynamics and morphodynamics of tidal channels, Ph.D. thesis, Università degli studi di Trento (2002).

[22] J. T. Kirby, G. Wei, Q. Chen, FUNWAVE 1.0, Fully Nonlinear Boussinesq Wave Model Documentation and User's Manual, Tech. Rep. CACR-98-06, University of Delaware (September 1998).

[23] G. Wei, J. T. Kirby, A. Sinha, Generation of waves in Boussinesq models using a source function method, Coast.Eng. 36 (1999) 271-299.

[24] M. A. Walkley, A numerical method for extended Boussinesq shallow-water wave equations, Ph.D. thesis, University of Leeds (1999).

[25] Y. Zhang, A. B. Kennedy, N. Panda, C. Dawson, J. J. Westerink, Generating-absorbing sponge layers for phase-resolving wave models, Coast.Eng. 84 (2014) 1-9.

[26] R. Cienfuegos, E. Barthelemy, P. Bonneton, A fourth-order compact finite volume scheme for fully nonlinear and weakly dispersive Boussinesq-type equations. part II: Boundary conditions and validation, Int.J.Numer.Meth.Fluids 53 (2007) 1423-1455.

[27] E. Wolanski, D. Williams, E. Hanert, The sediment trapping efficiency of the macro-tidal Daly Estuary, tropical Australia, Estuarine, Coastal and Shelf Science 69(1) (2006) 291-298. 
[28] J. C. Winterwerp, Z. B. Wang, A. van Braeckel, G. van Holland, F. Kosters, Man-induced regime shifts in small estuaries II: a comparison of rivers, Ocean Dynamics 63(11-12) (2013) 1293-1306.

[29] H. H. G. Savenije, E. J. M. Veling, Relation between tidal damping and wave celerity in estuaries, J. Geophys. Res. 101 (2005) doi:10.1029/2004JC002278.

[30] H. H. G. Savenije, A simple analytical expression to describe tidal damping or amplification, J. Hydrol. 243 (2001) 205-215.

[31] G. Vignoli, M. Toffolon, M. Tubino, Non-linear frictional residual effects on tide propagation, Proceedings of XXX IAHR Congress (2003) 291-298.

[32] H. Chanson, Tidal Bores, Aegir, Aegir, Eagre, Mascaret, Pororoca: Theory and Observations, World Scientific, Singapore, 2012. 